\title{
Design of Scale Meter Auto-Verification Time Optimization Model
}

\author{
Min Ren ${ }^{1}$, Qiqi Shu ${ }^{1}$, Le Yang ${ }^{1}$, Yabin $\mathrm{Ma}^{1}$, Letian Xie ${ }^{1}$, Jian Xu ${ }^{2}$ \\ 1. Metrology Center of State Grid Anhui Electric Power Co., Ltd., Hefei China \\ 2. NARI Technology Development Co., Ltd., Nanjing China
}

\begin{abstract}
With the concept of the State Grid Corporation of intensive management meter put forward and the completion of metering and intelligent provincial center of fully automated assembly line meter verification system in-depth applied, large scale power meter centralized verification challenges the intelligent verification process. So research on the optimization of watt-hour meter automatic verification time, and improved efficiency of the watt-hour meter verification is help for development of the meter intensive management model.
\end{abstract}

Keywords—meter; verification; waiting time; pulse interval

\section{INTRODUCTION}

With the rapid development of smart grid, the intensive management concept of electricity meters is proposed specifically, and the provincial measurement center has become the place for centralized verification of electricity meters [1]. At present, each provincial company has built an intensive and large-scale provincial-level measurement center, and uses the intelligent automatic pipeline electric energy meter verification system to seamlessly interface with the automated three-dimensional library system. The province unified meter verification, unified storage, unified distribution.

The measurement verification result is an important basis for the measurement accuracy of the electric energy meter and the evaluation of the effectiveness of other accuracy measurement methods. The verification efficiency of the electric energy meter determines whether it can meet the requirements of subsequent distribution and other links. Therefore, the process of energy metering verification and its cost have become the focus of analytical research $[2,3]$.

\section{Design of MeAsurement VerificAtion Method}

According to the regulations of JJG 596-2012 electronic ac energy meter, the electronic energy meter needs to detect as many as 86 error points, and the detection process of each error point includes the boost current, error point detection, and voltage and current return to zero. The total time required for the detection of 86 error points is 26 minutes, 78 minutes and 6 minutes, respectively. In addition, it takes 7 minutes for the computer to automatically switch between measurement items $[4,5]$. With the deepening of energy metering and intensification management, the multiplication of electric energy metering has brought great pressure to the energy meter verification system [6,7]. The cost of metrological verification includes manual input, asset investment and time investment.

The following is an example of a single-phase electronic active power meter (ammeter or test meter) to analyze the current metering verification method. The pulse constant $C_{\text {MUT }}$ tells the energy corresponding to a pulse (interval). So the number of pulses the meter outputs (within the range of accuracy levels allowed) reflects the amount of energy $\Delta E=1 \mathrm{Imp} / C_{\mathrm{MUT}}$ passing through the meter. The electronic energy meter usually outputs two kinds of pulses :(1) the light pulse, which is emitted by the LED on the electricity meter panel; (2) electric pulse sent by two end buttons in the terminal box of the meter.

The calibration of the meter involves the identification appearance inspection, pressure-resistant test, accuracy test and other items, among which the accuracy test includes (1) basic error test, (2) constant test, (3) starting test, and (4) latent test. The output pulse must be used. Accuracy testing requires the use of standard electricity meters (referred to as standard meters) and meter verification devices.

The pulse constant $C_{\mathrm{MS}}$ of the standard table is much larger than that of the tested table. It is usually assumed to be the actual value, but $C_{\mathrm{MUT}}$ is a nominal value.

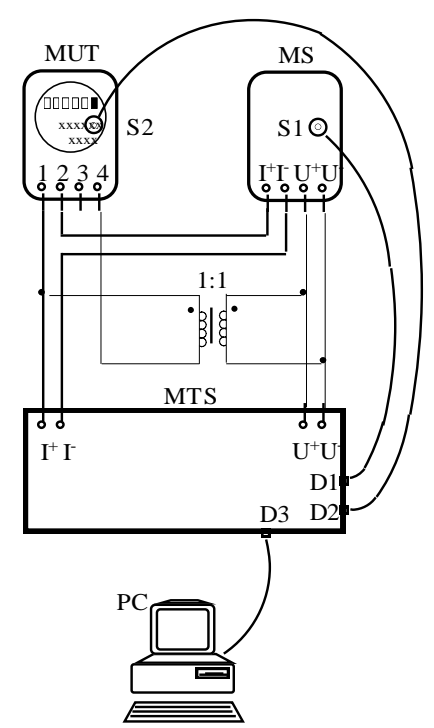

Fig.1 Single-phase electronic energy meter testing schematic 
The main functions of the verification device are :(1) provide stable power to the tested and standard tables; (2) collect and process the output pulse of the tested form and standard form, such as calculating the relative error of the tested form and the number of pulses of the timer.

In Fig.1, MUT is the tested table, MS is the standard table, MTS is the electricity meter verification device, and PC is the control computer. The MTS has a current input and output button I+I-, and the MUT and the MS are connected in series to the terminal button. The MTS has a voltage terminal U+U-, and the MUT connects the terminal in parallel with the MS through a 1:1 transformer. The pulse collectors S1, S2 are connected to the MTS through ports D1, D2. The PC controls the MTS through the D3 port.

\section{A. Basic error test}

The specifications of a model checked electric energy meter are shown in Tab.1.

Tab.1 A type of meter specifications

\begin{tabular}{ccccc}
\hline $\begin{array}{c}\text { Level of } \\
\text { accuracy }\end{array}$ & $\begin{array}{c}\text { Reference } \\
\text { voltage } U_{\mathrm{n}}\end{array}$ & $\begin{array}{c}\text { Reference } \\
\text { current } I_{\mathrm{b}}\end{array}$ & $\begin{array}{c}\text { Maximum } \\
\text { current } I_{\max }\end{array}$ & $\begin{array}{c}\text { Constant } \\
C_{\mathrm{MUT}}\end{array}$ \\
\hline 2 & $220 \mathrm{~V}$ & $5 \mathrm{~A}$ & $60 \mathrm{~A}$ & $1200 \mathrm{imp} / \mathrm{kWh}$ \\
\hline
\end{tabular}

The electric energy meter verification device calculates the relative error of the checked table according to the following formula (1).

$$
e=\frac{M_{\mathrm{MS}}^{*}-M_{\mathrm{MS}}}{M_{\mathrm{MS}}} \times 100 \%
$$

In the formula (1), $M_{\mathrm{MS}}$ indicates the number of pulses actually issued by the standard table in the $M_{\text {MUT }}$ pulse intervals of the test meter. $M_{\mathrm{MS}}^{*}$ denotes the number of standard table pulses calculated by $C_{\mathrm{MS}}$ and $C_{\mathrm{MUT}}$ in the same time interval, so that $M_{\mathrm{MS}}^{*}=\frac{C_{\mathrm{MS}}}{C_{\mathrm{MUT}}} M_{\mathrm{MUT}}$ can be obtained. Bring it into equation (1) to get:

$$
e=\left(\frac{C_{\mathrm{MS}}}{C_{\mathrm{MUT}}} \cdot \frac{M_{\mathrm{MUT}}}{M_{\mathrm{MS}}}-1\right) \times 100 \%
$$

The time to obtain the relative error according to the principle of formula (2) (only consider the time of collecting the pulse, ignoring the calculation time) is:

$$
T_{e}=M_{\mathrm{MUT}} T_{\mathrm{P}}(\mathrm{s})
$$

In the formula, $M_{\text {MUT }}$ is selected according to needs. The larger the value, the longer the test time, but the smaller the quantization error. The quantization error is:

$$
E=\frac{C_{\mathrm{MUT}}}{C_{\mathrm{MS}} M_{\mathrm{MUT}}} \times 100 \%
$$

Formula (4) refers to the pulse interval of the tested meter:

$$
T_{\mathrm{P}}=\frac{3.6 \times 10^{6}}{U \cdot I \cdot \cos \phi \cdot C_{\mathrm{MUT}}}(\mathrm{s})
$$

In formula (5), $U$ is the test voltage, which is usually fixed as the reference voltage $U_{\mathrm{n}} ; I$ is the test current, which varies from $0.05 I_{\mathrm{b}}$ to $I_{\text {max }}$; $\cos \phi$ is power factor, 1 or $0.5 \mathrm{~L}$. The pulse intervals $T_{\mathrm{P}}$ of the tested table of specifications under various load conditions are shown in Tab.2.

\begin{tabular}{c|c} 
Tab.2 The specifications meter pulse interval of each load \\
\hline $\begin{array}{c}\text { Load conditions } \\
(U, I, \cos \phi)\end{array}$ & Pulse interval $\left(T_{\mathrm{P}}\right)$ \\
\hline$U_{\mathrm{n}}, 0.05 I_{\mathrm{b}}, 1$ & $54.6 \mathrm{~s}$ \\
\hline$U_{\mathrm{n}}, 0.1 I_{\mathrm{b}}, 1$ & $27.3 \mathrm{~s}$ \\
\hline$U_{\mathrm{n}}, I_{\mathrm{b}}, 1$ & $2.7 \mathrm{~s}$ \\
\hline$U_{\mathrm{n}}, 0.5 I_{\max }, 1$ & $0.2 \mathrm{~s}$ \\
\hline$U_{\mathrm{n}}, I_{\max }, 1$ & $0.1 \mathrm{~s}$ \\
\hline$U_{\mathrm{n}}, 0.1 I_{\mathrm{b}}, 0.5 \mathrm{~L}$ & $54.6 \mathrm{~s}$ \\
\hline$U_{\mathrm{n}}, 0.2 I_{\mathrm{b}}, 0.5 \mathrm{~L}$ & $27.3 \mathrm{~s}$ \\
\hline$U_{\mathrm{n}}, I_{\mathrm{b}}, 0.5 \mathrm{~L}$ & $5.5 \mathrm{~s}$ \\
\hline$U_{\mathrm{n}}, 0.5 I_{\max }, 0.5 \mathrm{~L}$ & $0.5 \mathrm{~s}$ \\
\hline$U_{\mathrm{n}}, I_{\max }, 0.5 \mathrm{~L}$ & $0.2 \mathrm{~s}$ \\
\hline
\end{tabular}

\section{B. Starting test}

The verification method of starting test is as follows: voltage line load reference voltage $U_{\mathrm{n}}$, current line load start current $I_{\text {start }}$, power factor $\cos \phi=1$. MTS is required to receive at least one pulse within the specified time limit, otherwise MUT is unqualified.

The measurement verification regulations specify the calculation method for determining $T_{\text {start }}$ :

$$
T_{\text {start }}=1.2 \frac{60 \times 10^{3}}{U_{\mathrm{n}} \cdot I_{\text {start }} \cdot C_{\mathrm{MUT}}}(\mathrm{min})
$$

For the MUT of Tab.1 specification, So can calculate $I_{\text {start }}=0.005 I_{\mathrm{b}}=0.025 \mathrm{~A}$, substitute it into formula (6) and get $T_{\text {start }}=10.9 \mathrm{~min}$.

If the formula (6) specified in the regulations is not taken into account, it is assumed that the MUT keeps accurate measurement in the starting current $I_{\text {start }}$, then there should be pulse output within the time interval determined by formula (6). But actually the meter maker does not claim to have good metering characteristics under (tiny) starting current conditions, therefore, formula (5) times expansion factor $K$ (usually taking $K=1.2$ ) and let $\cos \phi=1$ estimate the interval of starting test impulse.

$$
T_{\mathrm{P}}^{\prime}=K \frac{3.6 \times 10^{6}}{U_{\mathrm{n}} \cdot I_{\text {start }} \cdot C_{\mathrm{MUT}}}(\mathrm{s})
$$


Therefore, it is possible to compare equations (6) and (7) to arrive at conclusion $T_{\text {start }}=T_{\mathrm{p}}^{\prime}$, that is, on the premise that MUT maintains accurate measurement in the starting current $I_{\text {start }}$, the estimated pulse interval of the meter start test is equal to the meter start test time.

\section{Creep test}

The method for verification of the submerged test is: the voltage line carries $1.15 U_{n}$, while the current line does not carry current. Within the prescribed time limit $T_{\text {no-load }}$, if MTS receives no more than one pulse, MUT is qualified; otherwise, it is deemed not qualified. And the reason why it's considered qualified to receive one pulse is that it's possible that MUT's $\Delta W$ is almost zero.

According to the regulations, formula $T_{\text {no-load }}$ is determined as formula (8) :

$$
T_{\text {no-load }}=\frac{480 \times 10^{6}}{U_{\mathrm{n}} \cdot I_{\max } \cdot C_{\mathrm{MUT}}}(\min )
$$

For the MUT of Tab.1 specification, So can calculate $T_{\text {no-load }}=30.3 \mathrm{~min}$.

\section{VERIFICATION TEST Time OPtIMIZATION MODEL}

\section{A. Basic error test}

$N$ power meters are tested at the same time. Since the output pulses of each meter have successively, the test time is the sum of the waiting time $T(N)$ (the time for the meter to be energized until the final output pulse has pulse output) and the error measurement time $T_{e}$ of the last meter.

Starting from the output power of MTS, the moment when the first pulse is output from the test meter $\operatorname{MUT}_{i}(i=1, \Lambda, N)$ is a random variable $T_{i}(i=1, \Lambda, N)$. This article uses the following two hypothesis.

Hypothesis 1: $T_{i}$ obeys the uniform distribution on $\left[0, T_{\mathrm{P}}\right]$.

Hypothesis 2: $T_{i}$ is independent.

From hypothesis 1, the distribution function of the waiting time $T_{i}$ of a single energy meter is:

$$
F_{T_{i}}(t)=\left\{\begin{array}{l}
0, t \subset(-\infty, 0] \\
\frac{t}{T_{\mathrm{P}}}, t \subset\left(0, T_{\mathrm{P}}\right] \\
1, t \subset\left(T_{\mathrm{P}},+\infty\right)
\end{array}\right.
$$

According to the definition of $T(N)$ :

$$
\begin{gathered}
T(N)=\max \left(T_{i}\right) \\
P\{T(N) \leq t\}=P\left\{\max \left(T_{i}\right) \leq t\right\}=P\left\{\prod_{i=1}^{N} T_{i} \leq t\right\}
\end{gathered}
$$

From the above formula (11) and hypothesis 2 , it can be known that: $P\{T(N) \leq t\}=\prod_{i=1}^{N} P\left\{T_{i} \leq t\right\}$. Therefore, the distribution function of $T(N)$ is:

$$
F_{T(N)}(t)=\left\{\begin{array}{l}
0, t \subset(-\infty, 0] \\
\frac{t^{N}}{T_{\mathrm{P}}^{N}}, t \subset\left(0, T_{\mathrm{P}}\right] \\
1, t \subset\left(T_{\mathrm{P}},+\infty\right)
\end{array}\right.
$$

The probability density function of $T(N)$ is:

$$
f_{T(N)}(t)=\frac{d F_{T(N)}(t)}{d t}=\left\{\begin{array}{c}
N \frac{t^{N-1}}{T_{\mathrm{P}}^{N}}, t \subset\left(0, T_{\mathrm{P}}\right] \\
0,
\end{array}\right.
$$

The expectation of $\mathrm{A}$ can be calculated according to formula (12) and formula (13) :

$$
E\{T(N)\}=\int_{-\infty}^{+\infty} t d F_{T(N)}(t)=\frac{N T_{\mathrm{P}}}{N+1}
$$

As can be seen from formula (12) and (13), as the number of meters $N$ increases, the waiting time is almost certainly very close to the pulse interval time $T_{\mathrm{P}}$ of meters.

This is also indicated by the probability density of $T(N)$ in formula (13), as shown in Fig.2.

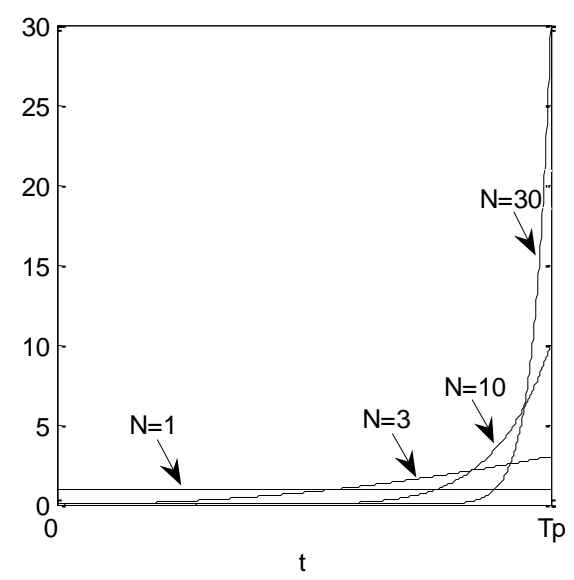

Fig.2 Probability density of the waiting time

Specifically, when $N$ is 30 , the average waiting time is $0.968 T_{\mathrm{P}}$, and the probability of waiting time greater than $0.9 T_{\mathrm{P}}$ is 0.958 . The waiting time for meter verification is 
infinitely close to $T_{\mathrm{P}}$ for the material verification of single 60 -meter bit power meter in the current intelligent verification pipeline system. Thus, the total waiting time of the basic error test can be obtained by summing the numbers in the second column of Tab.2.

\section{B. Starting test}

The start test of the meter is similar to the basic error test. When the number of the meter is $N \geq 30$, the start test time is almost certainly very close to a pulse interval time $T_{\mathrm{P}}^{\prime}$.

\section{Creep test}

It can be seen from the above that the power meter creep test time $T_{\text {no-load }}$ is the minimum pulse interval allowed under the creep test condition.

\section{CONCLUSION}

In this paper, the centralized calibration method of electric energy meter in the intensive management mode of provincial metrology center is analyzed. For the batch verification of the quantity of electric energy meter, the following conclusion can be drawn: the waiting time of the basic error test (the load point specified in Tab.2) is about $173 \mathrm{~s}$, the start test time was $10.9 \mathrm{~min}$ and the creep test time was $30.3 \mathrm{~min}$.This paper provides a reference for the optimization of the calibration time and the improvement of work efficiency of the batch electric energy meter.

\section{REFERENCES}

[1] Yan Qiang, et al. Research and Design of Production Management System for Power Measurement Verification[J]. China Measurement technology, 2003, 1(10):37-39.

[2] Zhang Yan, et al. Research and Application of Intelligent Verification Line System for Electric Energy Meter[J]. Electrical Measurement \& Instrumentation, 2009, 46(528):74-77.

[3] Fu Zhichao, Geng Xinzhi, Wang Lunzhan, et al .The New Requirments to Calibration System of Static Multi-meter for Energy(to be continued)[J] .Electrical Measurement \& Instrumentation, 2004, 41(7):13.

[4] JJG 596-2012 Electronic Energy Meter Verification Procedure [S].

[5] Q/SCG-2010 Technical Specification for Single-phase Electronic Wattmeter [Z].

[6] Wang Yong, Lv Hua , Li Yequan, et al .Ameliorator of the Problem Found in the Test of the Calibrator for Watt-hour meter[J]. Electrical Measurement \& Instrumentation, 2003, 40(1):48-50, 57.

[7] Chen Yinglin , Huang Dexiang. Digital Watt-hour Metering System and Verification Equipment[J]. Electric Power Automation Equipment, 2009, 4(29):114-117. 